\title{
Aspectual composition in Modern Greek
}

\author{
Maria Flouraki \\ Department of Language and Linguistics, University of Essex, UK \\ https://doi.org/10.36505/ExLing-2006/01/0026/000026
}

\begin{abstract}
The rich aspectual system in Modern Greek involves both morphologically ex-pressed grammatical aspect and eventuality types. Particular emphasis is paid to the interaction between grammatical aspect and eventuality types, since it is due to this interaction that the verbal predicate acquires distinct meanings. The main aim of this paper is to explain potential changes in the meaning of the eventualities caused by the interaction with grammatical aspect and provide a formal analysis of this inter-action. I propose an analysis within Head-Driven Phrase Structure Grammar (HPSG), using Minimal Recursion Semantics (MRS) for the semantic representa-tions. I argue that grammatical aspect is a function which takes as arguments par-ticular eventualities. When the arguments are different from the required ones, then there are instances of reinterpretations, which are not instances of ungrammaticality. This can be explained with the introduction of subeventual templates, where gram-matical aspect combines with eventuality types and selects eventualities or subeventualities appropriate to its selection restrictions, using information that is already there in the denotation of the eventualities.
\end{abstract}

\section{Introduction}

Aspectual composition occurs when grammatical aspect (perfective and imperfective) and eventuality types (accomplishments, achievements, processes, states) carried by the verb along with its arguments combine to trigger particular meanings. This aspectual composition may change the denotation of the eventuality type resulting to aspectual shifts. (Moens and Steedman 1988, Jackendoff 1990, Pustejovsky 1995, Krifka 1998, de Swart 1998, Giannakidou 2002, Egg 2002, Michaelis 2004).

An instance of this phenomenon is found in Modern Greek (M.G.) where there is a contrast between perfective and imperfective aspect, being overt in the morphology of the verb. The information, grammatical aspect presents, is affected by the eventuality type it combines with, which is implicit in the meaning of the verb phrase.

In (1) there is a process eventuality, which denotes a situation where Giannis loves Anna but is not clear when this loving situation starts and when and whether it finishes. When this eventuality occurs with imperfective aspect in (1a), it gets the default meaning of the eventuality, where no culmination point is denoted and no visible endpoints. In (1b) the same eventuality combines with perfective aspect, which may focus either on the

ExLing 2006: Proceedings of 1st Tutorial and Research Workshop on Experimental Linguistics, 28-30 August 2006, Athens, Greece 
initial stages of the eventuality in which case it acquires an inchoative reading or simply adds both endpoints, in which case we get a bounded reading.

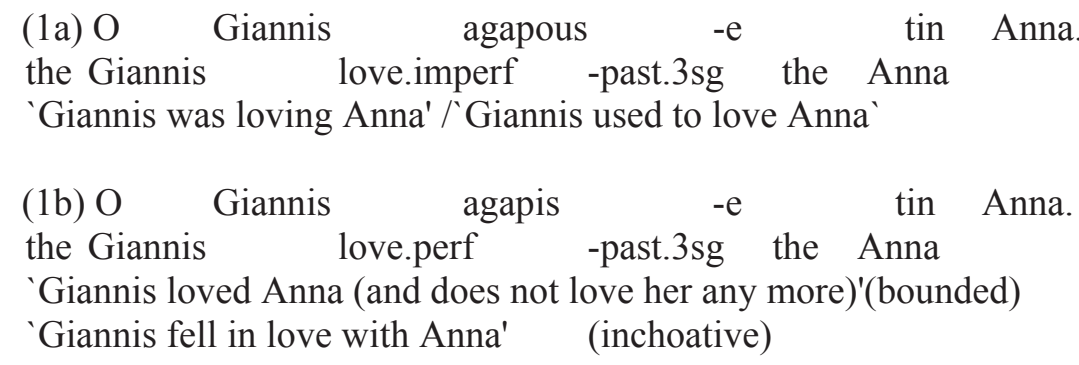

The aspectual shifts involved are subtypes of type shifts, which in the literature are formalised with the usage of a functor argument relation: $f(a)$, where $f$ is the functor and $a$ the argument. In the case of aspectual shifts, there is a functor-argument relation between grammatical aspect and eventuality types (2a). Aspect is further instantiated into the imperfective functor which combines with processes and states $(2 b)$.

(2a) aspect (eventuality type)

(2b) imperfective(process $\vee$ state)

There are cases where the argument is not the appropriate input for the functor as in (1b). However, there is no ungrammaticality involved but just reinterpretations occur, which remedy the conflict.

An explanation for these reinterpretations lies in the sphere of extralinguistic knowledge. The general relation $f(O p(a))$ is used, where the operator $O p$ added, is given by pragmatic context. A major drawback of these approaches is that these operators can not be appropriately constrained, so that they occur only where and when needed.

Following Michaelis (2004) and Pustejovsky (1995), we provide an alternative, where we develop a highly constructed inventory of eventuality types, which consists of eventualities as well as their subeventualities. These interact with grammatical aspect, which adds or selects the whole or subparts of the eventualities according to its selection restrictions.

\section{The Analysis}

The analysis proposed follows the framework of Head-Driven Phrase Structure Grammar (HPSG) (Pollard and Sag 1994), using Minimal Recursion Semantics (MRS) for the semantic representations (Copestake et al. 2000). Following (MRS) architecture, we introduce a number of relations, which represent both the grammatical aspect functor and the eventuality type argument given in (2a). 
The aspect-rel has the features L(a)B(e)L and BINDS as indicated in (3). The LBL identifies the relation and shows its scopal connection with the other relations whereas the BINDS feature shows the eventuality the aspectrel has to bind with. The aspect-rel combines with an eventuality through the BINDS feature and gives back the same or a different eventuality through the EVENT-STR(ucture) feature.
(3) $[$ aspect-rel
LBL handle
EVENT-STR
event-str
(4) [eventuality-rel
LBL handle
EVENT-STR [EVENT1 process
EVENT2 state
BINDS event-str RESTR $1<2]$ ]

The eventualities are decomposed into subparts so as grammatical aspect to be able to select the appropriate subpart in each case. Following Pustejovsky (1995), we support that each eventuality-rel has an event structure (EVENT-STR), whose value is a feature structure, that consists of different subevententualities indicated by the features EVENT1 and EVENT2.

The eventuality-rel in (4) introduces apart from the attributes LBL, the attribute EVENT-STR, which has two subeventualities: An EVENT1 with value a process type and an EVENT2 with value the state type. Their temporal ordering is guaranteed through the RESTR(iction) attribute, which states that there is a precedence temporal relation between EVENT1 and EVENT2.

Following Michaelis 2004, we support that as in the Romance languages, imperfective aspect in M.G. is a type-selecting operator, which reflects the eventuality type of its arguments. It modulates when it is necessary the aspectual properties of its argument and denotes eventuality types and place constraints upon the types it combines with. This kind of combination is guaranteed by the Aktionsart preservation principle, where no extra material is needed intervene in the functor argument relation.

Hence, the imperfective functor takes as argument particular eventualities and when the argument is not the appropriate input, the functor selects or adds a subpart to the eventuality it combines with. It selects process eventualities and returns an output of the same eventuality as the input as in (5a). When it combines with transition eventualities, it selects only the process subeventuality which is appropriate for its selection type as it is shown in (5b), where when the input is a transition then the output is just the process subeventuality.

(5) $F_{\text {imperf }}(X, Y)=Z$

(5b) if $\mathrm{Y}=[\mathrm{EVENT} 1$ process $]$, then $\mathrm{Z}=\mathrm{Y}$ [EVENT1 process]

$(5 \mathrm{c})$ if $\mathrm{Y}=[\mathrm{EVENT} 1$ process

EVENT2 state], then $\mathrm{Z}=[\mathrm{EVENT} 1$ process $]$ 


\section{Conclusion}

Through the account provided we have shown that eventualities consist of subeventual templates and grammatical aspect selects each time an appropriate subeventuality as input according to its selectional restrictions. Particular meanings are inferred which are already there in the denotation of the eventuality and they just need to be picked up by grammatical aspect.

\section{Acknowledgements}

I would like to thank my PhD supervisor Prof. Louisa Sadler and Dr. Doug Arnold. This research was supported by ESRC funding. Contact details: maria.flouraki@gmail.com

\section{References}

Copestake, A., Flickinger D., Pollard C. and Sag, I. A. 2000. Minimal Recursion Semantics: an Introduction. Language and Computation 1, vol.3, 1-47.

De Swart, H. 1998. Aspect shifts and Coercion. Natural language and Linguistic Theory 16, 347-385

Egg, M. 2002. Semantic Construction for Reinterpretation Phenomena. Journal of Semantics 15(1), 37-82.

Giannakidou, A. 2002. A puzzle about Until and the present perfect. In Alexiadou, A., Rathert, M. and von Stechow, A. (eds.), Perfect explorations, Mouton de Gruyter.

Jackendoff, R. 1997. The Architecture of the Language Faculty. Cambridge, MA: MIT Press.

Krifka, M. 1998. The origins of Telicity. In Rothstein, S. (ed.), Events and Grammar, 197-235, Great Britain.

Michaelis, L. 2004. Type shifting in Construction Grammar: An integrated approach to Aspectual Coercion. Cognitive Linguistics 15, 1-67.

Moens, M, and Steedman, M. 1988. Temporal ontology and Temporal Reference. Computational Linguistics 14, 15-28.

Pollard, C. J. and Sag, I. A. 1994. Head-Driven Phrase Structure Grammar. Stanford: CSLI publications.

Pustejovsky, J. 1995. The Generative Lexicon. Cambridge, MA: MIT Press. 